\title{
Capacity Bounds for MIMO System in TWDP Fading Channel
}

\author{
Bhavnika Garg and Aman Singh ${ }^{\mathrm{b}, *}$ \\ ${ }^{a}$ Department of Electrical and Electronics Engineering, Lovely Professional University Phagwara, Punjab, India \\ ${ }^{b}$ Department of Computer Science and Engineering, Lovely Professional University Phagwara, Punjab, India
}

\begin{abstract}
Many statistical distributions including Rayleigh, Rician, Nakagami-m, Hoyt, $\eta-\mu$ and $\kappa-\mu$ are proposed in literature to model fading in wireless Communication systems. This paper presents the study of MIMO system in a recently proposed Two Wave with Diffuse Power (TWDP) fading model, having two specular multipath components in the presence of diffusely propagating waves. It has been verified that the TWDP fading model is better suited to represent real world frequency selective fading on the basis of data collected from wireless sensor networks. This paper studies the performance of MIMO system in TWDP fading scenario. Upper and lower bounds of the capacity for MIMO system have been derived in TWDP fading scenario.
\end{abstract}

Keywords: MIMO, massive MIMO, capacity, SNR, TWDP, Rayleigh, Rician.

(Submitted on March 22, 2017; Revised on May 27, 2017; Accepted on June 15, 2017)

(C) 2017 Totem Publisher, Inc. All rights reserved.

\section{Introduction}

Wireless industry has completely revolutionized the communication technology. It started with SISO (Single input and single output) system where one antenna at transmitter transmits and one antenna at receiver receives with no additional processing required. SISO although being the simplest system is not currently employed because of its capacity limitations imposed by interference and fading. SIMO systems overcome limitations of SISO where receiver diversity is present to combat the adverse effects of fading by receiving same signal from number of independent sources. SIMO although easy to implement requires processing at receiver end and offers problems of limited data rate, not enough for current wireless needs where data in form of texts, images and videos needs to be transferred with high speed. Some authors [1] then suggested use of MISO systems i.e. to employ transmit diversity, where receiver selects optimum signal and no processing is required. But, limited data rate (bits transmitted per second) still remained major issue, which came to an end with implementation of MIMO (multiple input and multiple output) systems. MIMO provides diversity at both sides i.e. both at transmitter and receiver, hence is able to combat interference and fading in an efficient way [1,2]. MIMO in fact, uses spatial diversity, random fading and multipath delay spread in its benefits and thus improves throughput and attains data rates that are required to meet today's needs [3].

Fading and interference are main characterizing features of any wireless scenario. Hence, study of any wireless system in a proper fading model that properly characterizes propagation channel is essential.

\footnotetext{
${ }^{*}$ Corresponding author. Tel.: +91-998-877-9907.

E-mail address: amansingh.x@gmail.com.
} 
The effect of fading's, such as Rayleigh, Rician, Nakagami etc. on wireless systems have already been published in many research papers. But, TWDP (Two Wave Diffuse Power) fading has still not been applied in MIMO and Massive MIMO systems. MIMO performs better when channel is highly faded or in other words when most severe form of fading exists. Also, the work in [4] shows that TWDP channel makes a link which is even worse than Rayleigh/Rician channel. Hence TWDP model should be considered to study performance of a wireless communication system. In this paper the performance of MIMO has been studied in TWDP fading channel and impact of TWDP fading parameters on MIMO's capacity is analysed.

\section{TWDP Fading Model}

In wireless communication, signal propagates through different paths and arrives at the receiver; hence received signal is combination of multipath waves which arrive with different phases and amplitudes. The propagating waves are of two types specular and diffuse. Specular waves are characterized by strong LOS component plus reflected components whereas diffuse waves are made up of faint waves with random magnitudes and phases commonly called as scattered components [5]. TWDP stands for Two Wave Diffuse Power fading. It has two dominant LOS components and other diffusely propagation waves. Authors in [6] first introduced it as the form of fading which exist because of use of directional antennas, wide band signals and typical narrow band receiver operations. TWDP fading or hyper Rayleigh fading mathematically models channels such as wireless signals propagating in aircrafts, cavity environments like airframes and shipping containers where wireless sensors are commonly deployed for security and maintenance issues. These environments give rise to frequency selective fading statistics which is more severe than Rayleigh fading. Hence a new worst case model is predicted for these applications. TWDP Model is hence used to model harsh environments which exhibit fading worse than Rayleigh channel fading [7]. A typical received signal in Wireless Communication is made up of $\mathrm{N}$ strong LOS components and M diffuse components as given in equation (1).

$$
V_{r}=\sum_{i=1}^{N} V_{i} \exp \left(j \emptyset_{i}\right)+\sum_{k=1}^{M} V_{i} \exp \left(j \emptyset_{k}\right) .
$$

As given in equation (1) and represented by fig.1, specular Component is a single term $\left\{V_{i} \exp \left(j \emptyset_{i}\right)\right\}$, representing one arriving multipath wave. The phase $\emptyset_{i}$ of a specular component is random, but the envelope $V_{i}$ is constant. A Non- specular component/diffuse component has numerous individual waves which carry power negligible to the total average power [8]. A typical TWDP fading model is shown in figure1.
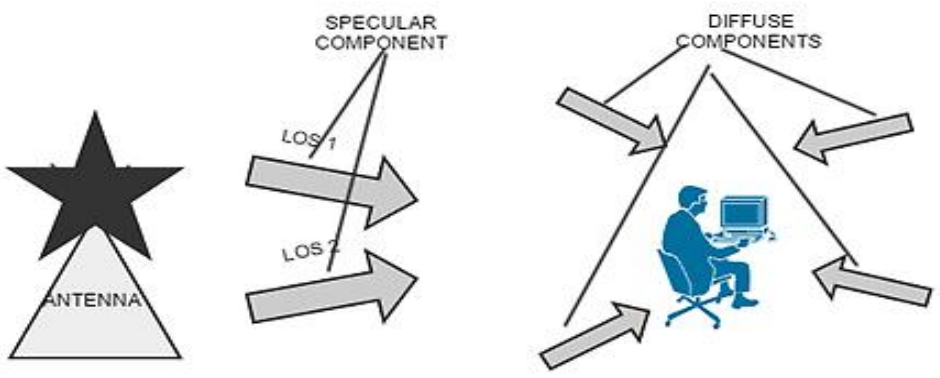

Figure 1. Illustration of SPECULAR and DIFFUSE components.

The received TWDP fading signal $V_{r}$ consists of two specular components and a diffuse component given by

$$
V_{r}=V_{1} \exp \left(j \emptyset_{1}\right)+V_{2} \exp \left(\mathrm{j} \emptyset_{2}\right)+\underline{\mathrm{X}+\mathrm{jY}}
$$

where, $V_{1}$ and $V_{2}$ are voltage amplitudes of two specular components with uniformly distributed phases $\emptyset_{1}$ and $\emptyset_{2} \sim U$ $(0,2 \pi)$. Diffuse components $\mathrm{X}, \mathrm{Y}$ are Gaussian distributed with zero mean and variance $\sigma^{2}$ [8]. Two parameters $\mathrm{K}$ and $\Delta$ characterize the pdf of TWDP fading where, $\mathrm{K}$ is ratio of the specular power to diffused power and $\Delta$ is relative strength of two specular components. These parameters are defined as follows

$$
\mathrm{K}=\frac{V_{1}^{2}+V_{2}^{2}}{2 \sigma^{2}} \quad \text { and } \quad \Delta=\frac{2 V_{1} V_{2}}{V_{1}^{2}+V_{2}^{2}},
$$

where, $2 \sigma^{2}$ represents the average power of the diffused waves [6]. 


\section{Performance Analysis of MIMO System in TWDP Fading Scenario}

Consider a MIMO system with $N_{t}$ transmit and $N_{r}$ receive antennas as shown in fig. 2 . Let $\mathbf{x} \in N_{t} \times 1$ is transmitted vector, $\mathbf{H} \in C^{N_{r} \times N_{t}}$ is channel matrix , and $\mathbf{n} \in N_{r} \times 1$ is noise vector and, $\mathbf{y} \in N_{r} \times 1$ is received vector, then MIMO system can be presented as below in the form of equation (4),

$$
\mathbf{y}=\mathbf{H x}+\mathbf{n},
$$
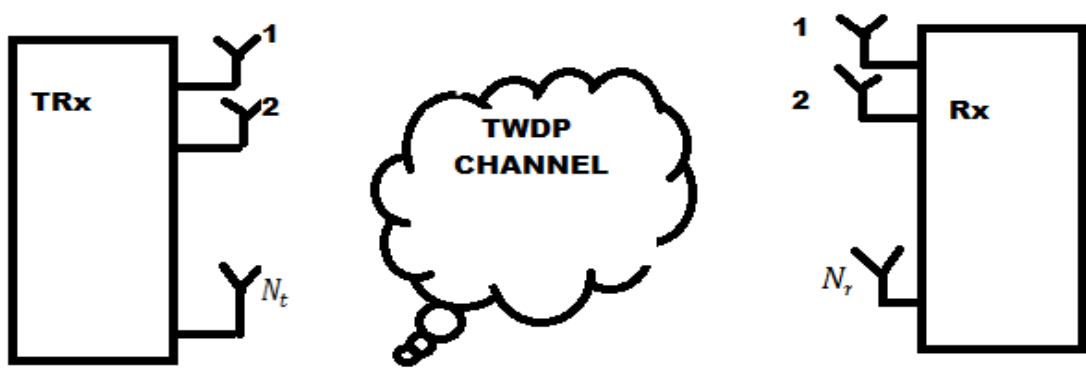

Fig. 2. MIMO system model.

Capacity of MIMO System is given by

$$
C_{M I M O}=\log _{2}\left(\operatorname{det}\left(I_{N_{r}}+\frac{\rho}{N_{t}} \boldsymbol{H H}^{H}\right)\right) .
$$

In equation (5) $\rho$ is SNR (signal to noise ratio).

However, when TWDP fading exists elements of $\boldsymbol{H}$ are considered to be TWDP faded, and capacity in TWDP fading is given by (6)

$$
C_{M I M O-T W D P}=\log _{2}\left(\operatorname{det}\left(I_{N_{r}}+\frac{\rho}{N_{t}} \boldsymbol{H}_{\text {TWDP }} \boldsymbol{H}_{\text {TWDP }}{ }^{H}\right)\right)
$$

In order to plot capacity, the TWDP fading scenario is first simulated in MATLAB. All comparisons are made in three fading scenario i.e. in Rayleigh, Rician and TWDP fading model. Rayleigh fading is random fading model, which is modelled in MATLAB using two normal random variables of zero mean and $\sigma^{2}$ variance.

$$
\boldsymbol{H}_{\text {Rayleigh }}=\operatorname{randn}\left(N_{r}, N_{r}\right)+\mathrm{j} * \operatorname{randn}\left(N_{r}, N_{t}\right)
$$

Rician fading has one LOS path, which states that one random variable is non- zero mean while other is zero mean both with $\sigma^{2}$ variance.

$$
\boldsymbol{H}_{\text {Rician }}=\left(\left(\operatorname{randn}\left(N_{r}, N_{r}\right) * \sigma\right)+\text { mean }\right)+\mathrm{j} * \operatorname{randn}\left(N_{r}, N_{r}\right) * \sigma
$$

In a TWDP (Two wave diffuse power) there are three components two components are deterministic and one is stochastic. Hence, TWDP fading is modelled in the same way as Rayleigh but the value of one random variable is altered by adding strength of two LOS components in the expression. The TWDP channel matrix is given as

$$
H_{T W D P}=\mathrm{aH} 1_{L O S}+\mathrm{bH} 2_{L O S}+\mathrm{c} H_{\text {Rayleigh }}
$$

where again $\mathrm{a}, \mathrm{b}$ and $\mathrm{c}$ are scalars ( $\mathrm{a}$ and $\mathrm{b}$ are powers of two LOS components and $\mathrm{c}$ is power of stochastic component). For TWDP fading $\mathrm{K}$-factor (ratio of the specular power to diffused power) and $\Delta$ factor (relative strength of two specular components) as defined in (3) can be expressed as

$$
K_{T W D P}=\frac{a^{2}+b^{2}}{c^{2}} \text { and } \Delta_{T W D P}=\frac{2 a b}{a^{2}+b^{2}}
$$


Here, $\mathrm{K}$ and $\Delta$ holds the same meaning as mentioned in (3), but are now expressed in form of scalars.

$\mathrm{K} \rightarrow 0$ channel is pure Rayleigh

$\mathrm{K} \rightarrow \infty$ and $\Delta=0$ channel is Rician

$\mathrm{K} \rightarrow \infty$ and $\Delta \rightarrow 1$ channel is TWDP

Solving (10) we get,

$$
\begin{aligned}
& 2 \mathrm{a}=\sqrt{K c^{2}+\Delta K c^{2}}+\sqrt{K c^{2}-\Delta K c^{2}} \\
& 2 \mathrm{~b}=\sqrt{K c^{2}+\Delta K c^{2}}-\sqrt{K c^{2}-\Delta K c^{2}}
\end{aligned}
$$

and a,b are strength of two LOS components and $\mathrm{c}$ is strength of diffuse components. The capacity of $4 \times 4$ MIMO system is plotted in different fading environments in figure 3.

Figure 3 shows that 4X4 MIMO performs excellently in TWDP channel as compared to other fading channels due to severest form of fading. At SNR of $25 \mathrm{~dB}$, TWDP channel attains highest capacity of 30bits/sec/hertz whereas it is 26bits/sec/hertz for Rayleigh channel and 24 bits/sec/hertz for Rician channel. This figure proves the fact given in [3].

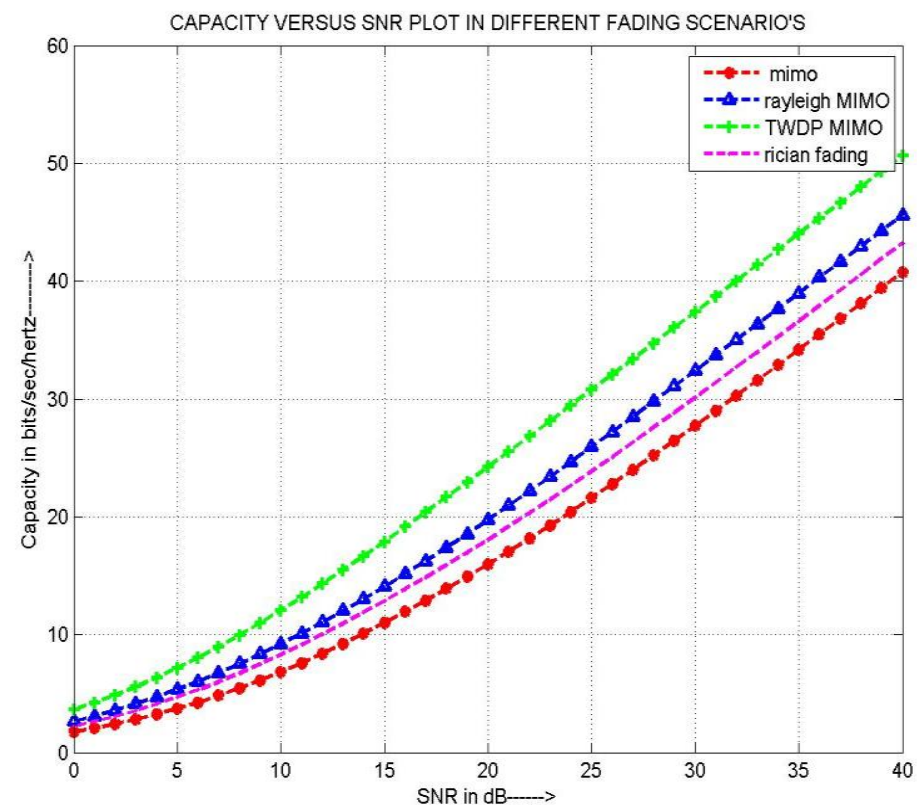

Figure 3. Performance of all fading scenario's in MIMO system.

\section{Bounds on Capacity of MIMO System in TWDP Fading}

The exact capacity expression of the MIMO system in TWDP channel depends on the Eigen values of the Hermitian matrix $\boldsymbol{H}_{\boldsymbol{T W D P}} \boldsymbol{H}_{\boldsymbol{T W D P}}^{H}$. Consider $\lambda_{i}, \mathrm{i}=1 \ldots \ldots \mathrm{n}$ be the eigen value of $\boldsymbol{H}_{\boldsymbol{T} W \boldsymbol{D} \boldsymbol{P}} \boldsymbol{H}_{\boldsymbol{T} W \boldsymbol{D} \boldsymbol{P}}^{H}$, then the capacity of MIMO system is given by

$$
C_{T W D P}=\sum_{i=1}^{N_{t}} \log _{2}\left(1+\frac{\rho}{N_{t}} \lambda_{i}\right)
$$

A. Lower capacity bound of MIMO system in TWDP fading

As TWDP system is made of three components, two LOS and one stochastic. Lower bound is achieved when both LOS parts being anti-phase cancel each other and only random part exists, which turns out to be same as Rayleigh. From equation (9) 


$$
H_{T W D P}=\mathrm{a} H 1_{L O S}+\mathrm{bH} 2_{L O S}+\mathrm{c} H_{\text {Rayleigh }}
$$

For calculating lower bound both LOS cancel each other, hence

$$
H_{T W D P}=H_{\text {Rayleigh }}
$$

Assume that, $N_{t}$ and $N_{r}=N$, then it can be shown [9,10] that for large $\mathrm{N}$ and large value of SNR, $\rho$, a capacity lower bound is given by

$$
C_{\text {Lower-Bound }}=\mathrm{N} * \log _{2}\left(\frac{\rho}{e}\right)
$$

where $\mathrm{e}$ is mathematical constant that is the base of natural logarithm.

B. Upper bound on capacity in TWDP fading

To derive upper bound of capacity of MIMO system in TWDP channel considers the TWDP channel matrix expression in terms of LOS and Rayleigh channel matrices. The product $\boldsymbol{H}_{T W D P} \boldsymbol{H}_{T W D P}^{H}$ ofTWDP channel matrix is given as

$$
\begin{aligned}
& H_{T W D P} H_{T W D P}^{H}=\left(\mathrm{a} H 1_{L O S}+\mathrm{bH} 2_{L O S}+\mathrm{cH} H_{\text {Ray }}\right) *\left(\mathrm{a} H 1_{L O S}+\mathrm{bH} 2_{L O S}+\mathrm{cH} H_{\text {Ray }}\right)^{H} \\
& =a^{2} H 1_{L O S} H 1_{\text {LOS }}^{H}+b^{2} H 2_{\text {Los }} H 2_{\text {LOS }}^{H}+c^{2} H_{\text {Ray }}+\mathrm{ab}\left(H 1_{\text {LOS }} H 2_{\text {LOS }}^{H}+H 2_{\text {Los }} H 1_{\text {LOS }}^{H}\right)+\operatorname{ac}\left(H \text { Ray } H 1_{\text {LOS }}^{H}+\right. \\
& \left.H 1_{L O S} H_{R a y}^{H}\right)+b c\left(H_{R a y} H 2_{L O S}^{H}+H 2_{L O S} H_{R a y}^{H}\right)
\end{aligned}
$$

From the expression given in equation (16), it is clear that capacity is sum of six components Pure LOS 1 , Pure LOS 2 , Pure Rayleigh term and three hybrid terms where, term with coefficient ab has two LOS components contributing jointly and terms containing coefficients ac and bc have both the LOS and Rayleigh components contributing together. Hence, taking statistical expectation of terms with ac and bc coefficients reduces to zero.

$$
\begin{gathered}
\mathrm{E}\left\{\mathrm{ac}\left(\boldsymbol{H}_{\text {Ray }} \boldsymbol{H} \mathbf{1}_{\text {Los }}^{H}+\boldsymbol{H} \mathbf{1}_{\text {Los }} \boldsymbol{H}_{\text {Ray }}^{H}\right)+\mathrm{bc}\left(\boldsymbol{H}_{\text {Ray }} \boldsymbol{H} \mathbf{2}_{\text {LOS }}^{H}+\boldsymbol{H} \mathbf{2}_{\text {Los }} \boldsymbol{H}_{\text {Ray }}^{H}\right)\right\} \\
=\mathrm{E}\left\{\mathrm{ac}\left(\boldsymbol{H}_{\text {Ray }} \boldsymbol{H} \mathbf{1}_{\text {Los }}^{H}+H \mathbf{1}_{\text {Los }} \boldsymbol{H}_{\text {Ray }}^{H}\right)\right\}+\mathrm{E}\left\{\mathrm{bc}\left(\boldsymbol{H}_{\text {Ray }} \boldsymbol{H} \mathbf{2}_{\text {Los }}^{H}+\boldsymbol{H} \mathbf{2}_{\text {Los }} \boldsymbol{H}_{\text {Ray }}^{H}\right)\right\}=0
\end{gathered}
$$

Now, equation (16) reduces to

$$
H_{T W D P} H_{T W D P}^{H}=a^{2} H 1_{L O S} H 1_{L O S}^{H}+b^{2} H 2_{L O S} H 2_{L O S}^{H}+c^{2} H_{R a y} H_{R a y}^{H} \text { abH } 1_{L O S} H 2_{L O S}^{H}+b a H 2_{L O S} H 1_{L O S}^{H}
$$

Considering, the two LOS components to be fully uncorrelated, hence putting terms with coefficients ab and ba to zero. Hence equation (17) reduces to

$$
H_{\text {TWDP }} H_{\text {TWDP }}^{H}=a^{2} H 1_{L O S} H 1_{L O S}^{H}+b^{2} H 2_{L O S} H 2_{L O S}^{H}+c^{2} H_{R a y} H_{R a y}^{H}
$$

thus, all hybrid terms contribution in capacity is neglected and only pure LOS and pure random components are considered in capacity expression as given in (19) below

$$
\begin{gathered}
C_{T W D P}=\log _{2} \operatorname{det}\left(\boldsymbol{I}_{N}+\frac{\rho}{N} a^{2} \boldsymbol{H} \mathbf{1}_{\text {Los }} \boldsymbol{H} \mathbf{1}_{\text {LOS }}^{H}+\frac{\rho}{N} b^{2} \boldsymbol{H} \mathbf{2}_{\text {Los }} \boldsymbol{H} \mathbf{2}_{\text {LOS }}^{H}+c^{2} \boldsymbol{H}_{\text {Ray }} \boldsymbol{H}_{\text {Ray }}^{H}\right) \\
=\sum_{i=1}^{N} \log _{2}\left(1+\frac{\rho}{N} \lambda_{i}\right),
\end{gathered}
$$

Here $\lambda_{i}$ are Eigen values of $a^{2} \boldsymbol{H} \mathbf{1}_{L \boldsymbol{O O S}} \boldsymbol{H} \mathbf{1}_{\text {Los }}^{H}+b^{2} \boldsymbol{H} \mathbf{2}_{\text {LOS }} \boldsymbol{H} \mathbf{2}_{\text {LOS }}^{H}+c^{2} \boldsymbol{H}_{\text {Ray }} \boldsymbol{H}_{\text {Ray }}^{H}$.

Determinant term in equation (19) is:

$$
\operatorname{det}\left(\boldsymbol{I}_{\boldsymbol{N}}+\frac{\rho}{N} a^{2} \boldsymbol{H} \mathbf{1}_{\text {LOS }} \boldsymbol{H} \mathbf{1}_{\text {LOS }}^{H}+\frac{\rho}{N} b^{2} \boldsymbol{H} \mathbf{2}_{\text {LOS }} \boldsymbol{H} \mathbf{2}_{\text {LOS }}^{H}+\frac{\rho}{N} c^{2} \boldsymbol{H}_{\text {Ray }} \boldsymbol{H}_{\text {Ray }}^{H}\right)
$$

Using property of matrices, in equation (21),

If $\mathbf{A}$ and $\mathbf{B}$ are respectively $\mathrm{N} \times \mathrm{M}$ and $\mathrm{M} \times \mathrm{N}$ matrices, then $\operatorname{det}\left\{\boldsymbol{I}_{\boldsymbol{N}}+\mathbf{A B}\right\}=\operatorname{det}\left\{\boldsymbol{I}_{\boldsymbol{M}}+\mathbf{B} \mathbf{A}\right\}$ 


$$
\begin{aligned}
& =\operatorname{det}\left\{\boldsymbol{I}_{\boldsymbol{N}}+\left[\sqrt{\frac{\rho}{N}} a \boldsymbol{H} \mathbf{1}_{\text {LOS }} \sqrt{\frac{\rho}{N}} b \boldsymbol{H} \mathbf{2}_{\text {LOS } \sqrt{\frac{\rho}{N}} c \boldsymbol{H}_{\text {Ray }}}\right]\left[\begin{array}{c}
\sqrt{\frac{\rho}{N}} a \boldsymbol{H} \mathbf{1}_{\text {LOS }}^{\boldsymbol{H}} \\
\sqrt{\frac{\rho}{N}} b \boldsymbol{H} \mathbf{2}_{\text {LOS }}^{\boldsymbol{H}} \\
\sqrt{\frac{\rho}{N}} c \boldsymbol{H}_{\text {Ray }}^{\boldsymbol{H}}
\end{array}\right]\right\} \\
& =\operatorname{det}\left\{\boldsymbol{I}_{3 N}+\left[\begin{array}{l}
\sqrt{\frac{\rho}{N}} a H \mathbf{1}_{\text {LOS }}^{H} \\
\sqrt{\frac{\rho}{N}} b \boldsymbol{H} \mathbf{2}_{\text {LOS }}^{\boldsymbol{H}} \\
\sqrt{\frac{\rho}{N}} c \boldsymbol{H}_{\text {Ray }}^{\boldsymbol{H}}
\end{array}\right]\left[\sqrt{\frac{\rho}{N}} a \boldsymbol{H} \mathbf{1}_{\text {LOS } \left.\sqrt{\frac{\rho}{N}} b H 2_{\text {LOS }} \sqrt{\frac{\rho}{N}} c \boldsymbol{H}_{\text {Ray }}\right]}\right\}\right.
\end{aligned}
$$

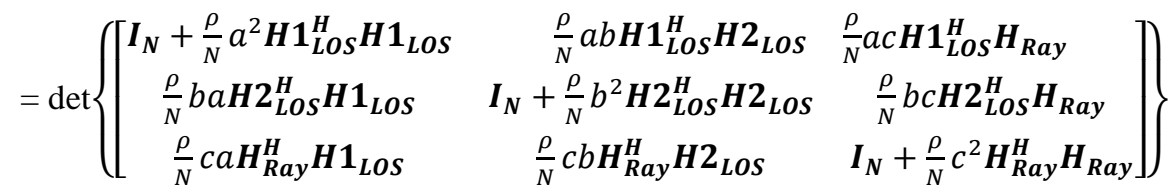

Now, terms with coefficients ab and ba can be put to zero in (25) as there is no correlation among the two LOS components. Hence equation (25) reduces to

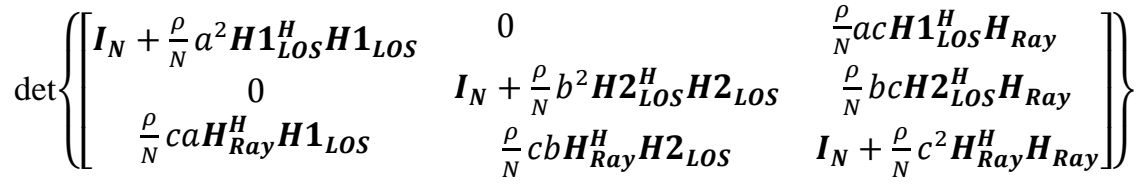

Now, the matrix is Hermitian and can be factorized using block lower - Diagonal -Upper (LDU) as follows

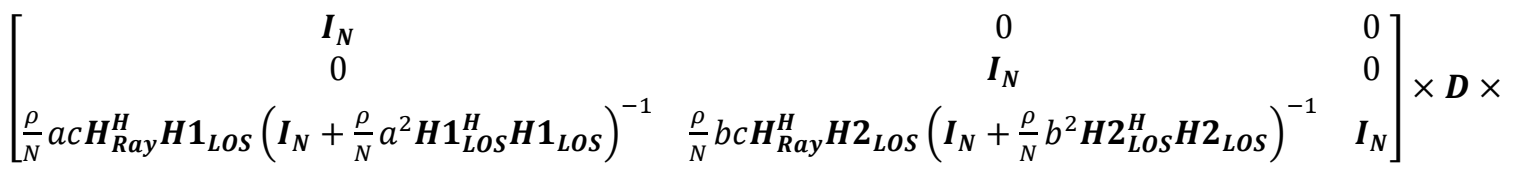

$$
\begin{aligned}
& {\left[\begin{array}{ccc}
\boldsymbol{I}_{N} & 0 & \left(I_{N}+\frac{\rho}{N} a^{2} \boldsymbol{H} \mathbf{1}_{L O S}^{H} \boldsymbol{H} \mathbf{1}_{L O S}\right)^{-1} \frac{\rho}{N} a c H 1_{L O S}^{H} H_{\text {Ray }} \\
0 & \boldsymbol{I}_{N} & \left(I_{N}+\frac{\rho}{N} b^{2} \boldsymbol{H} 2_{L O S}^{H} \boldsymbol{H} 2_{L O S}\right)^{-1} \frac{\rho}{N} b c H 2_{L O S}^{H} H_{\text {Ray }} \\
0 & 0 & \boldsymbol{I}_{N}
\end{array}\right]}
\end{aligned}
$$

Where $\mathbf{D}$ can be written as

$$
\mathrm{D}=\left[\begin{array}{ccc}
\boldsymbol{I}_{\boldsymbol{N}}+\frac{\rho}{N} a^{2} \boldsymbol{H} \mathbf{1}_{\text {LOS }}^{\boldsymbol{H}} \boldsymbol{H} \mathbf{1}_{L O S} & 0 & 0 \\
0 & \boldsymbol{I}_{N}+\frac{\rho}{N} b^{2} \boldsymbol{H} \mathbf{2}_{\text {LOS }}^{H} \boldsymbol{H} \mathbf{2}_{L O S} & 0 \\
0 & 0 & Z
\end{array}\right]
$$

Term, $\mathrm{Z}$ in Equation (28) is large and is given below in (29)

$$
\begin{aligned}
& \left\{\boldsymbol{I}_{\boldsymbol{N}}+\frac{\rho}{N} c^{2} \boldsymbol{H}_{\text {Ray }}^{\boldsymbol{H}} \boldsymbol{H}_{\text {Ray }}-\left(\frac{\rho}{N}\right)^{2} a^{2} c^{2} \boldsymbol{H}_{\text {Ray }}^{\boldsymbol{H}} \boldsymbol{H} \mathbf{1}_{L O S}\left(\boldsymbol{I}_{\boldsymbol{N}}+\frac{\rho}{N} a^{2} \boldsymbol{H} \mathbf{1}_{L O S}^{\boldsymbol{H}} \boldsymbol{H} \mathbf{1}_{L O S}\right)^{-1} \boldsymbol{H} \mathbf{1}_{L O S}^{H} \boldsymbol{H}_{\text {Ray }}-\left(\frac{\rho}{N}\right)^{2} b^{2} c^{2} \boldsymbol{H}_{\text {Ray }}^{\boldsymbol{H}} \boldsymbol{H} \boldsymbol{2}_{L O S}\left(\boldsymbol{I}_{\boldsymbol{N}}+\right.\right. \\
& \left.\left.\frac{\rho}{N} b^{2} H 2_{L O S}^{H} H 2_{L O S}\right)^{-1} H 2_{L O S}^{H} H_{R a y}\right\}
\end{aligned}
$$


Since the determinant of a block diagonal matrix is the product of the determinants of the diagonal blocks, using property of the determinant of a product of matrices

$$
\begin{aligned}
& \operatorname{det}\left\{\left[\begin{array}{ccc}
\boldsymbol{I}_{N}+\frac{\rho}{N} a^{2} \boldsymbol{H} \mathbf{1}_{L O S}^{\boldsymbol{H}} \boldsymbol{H} \mathbf{1}_{L O S} & 0 & \frac{\rho}{N} a c H \mathbf{1}_{L O S}^{\boldsymbol{H}} \boldsymbol{H}_{\text {Ray }} \\
0 & \boldsymbol{I}_{N}+\frac{\rho}{N} b^{2} \boldsymbol{H} \mathbf{2}_{L O S}^{\boldsymbol{H}} \boldsymbol{H} 2_{L O S} & \frac{\rho}{N} b c H 2_{L O S}^{\boldsymbol{H}} \boldsymbol{H}_{\text {Ray }} \\
\frac{\rho}{N} c a \boldsymbol{H}_{\text {Ray }}^{\boldsymbol{H}} \boldsymbol{H} \mathbf{1}_{L O S} & \frac{\rho}{N} c b \boldsymbol{H}_{\text {Ray }}^{\boldsymbol{H}} \boldsymbol{H} \mathbf{2}_{L O S} & I_{N}+\frac{\rho}{N} c^{2} \boldsymbol{H}_{\text {Ray }}^{\boldsymbol{H}} \boldsymbol{H}_{\text {Ray }}
\end{array}\right]\right\} \\
& \text { can be reduced to: }=1 \times \operatorname{det}\{\mathbf{D}\} \times 1 \\
& =\operatorname{det}\left\{\boldsymbol{I}_{\boldsymbol{N}}+\frac{\rho}{N} a^{2} \boldsymbol{H} \mathbf{1}_{\boldsymbol{L} \boldsymbol{O} \boldsymbol{S}}^{\boldsymbol{H}} \boldsymbol{H} \mathbf{1}_{\text {Los }}\right\} \times \operatorname{det}\left\{\boldsymbol{I}_{\boldsymbol{N}}+\frac{\rho}{N} a^{2} \boldsymbol{H} \mathbf{1}_{\boldsymbol{L} \boldsymbol{O} \boldsymbol{S}}^{\boldsymbol{H}} \boldsymbol{H} \mathbf{1}_{\boldsymbol{L O S}}\right\} \times \operatorname{det}\{\mathrm{Z}\}
\end{aligned}
$$

Now, $\mathrm{Z}$ can be solved as follows $\left\{\boldsymbol{I}_{\boldsymbol{N}}+\frac{\rho}{N} c^{2} \boldsymbol{H}_{\text {Ray }}^{\boldsymbol{H}} \boldsymbol{H}_{\text {Ray }}-\left(\frac{\rho}{N}\right)^{2} a^{2} c^{2} \boldsymbol{H}_{\mathrm{Ray}}^{\boldsymbol{H}} \boldsymbol{H} \mathbf{1}_{L \boldsymbol{L O S}}\left(\boldsymbol{I}_{\boldsymbol{N}}+\right.\right.$ $\left.\left.\frac{\rho}{N} a^{2} H 1_{L O S}^{\boldsymbol{H}} \boldsymbol{H} \mathbf{1}_{L O S}\right)^{-1} \boldsymbol{H} \mathbf{1}_{\text {LOS }}^{\boldsymbol{H}} \boldsymbol{H}_{\text {Ray }}-\left(\frac{\rho}{N}\right)^{2} b^{2} c^{2} \boldsymbol{H}_{\text {Ray }}^{\boldsymbol{H}} \boldsymbol{H} \mathbf{2}_{L O S}\left(\boldsymbol{I}_{\boldsymbol{N}}+\frac{\rho}{N} b^{2} \boldsymbol{H} \mathbf{2}_{\text {LOS }}^{\boldsymbol{H}} \boldsymbol{H} \mathbf{2}_{L O S}\right)^{-1} \boldsymbol{H} \mathbf{2}_{\text {LOS }}^{\boldsymbol{H}} \boldsymbol{H}_{\text {Ray }}\right\}$

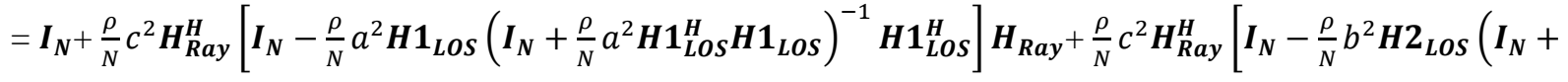

$$
\begin{aligned}
& \left.\left.\frac{\rho}{N} b^{2} \boldsymbol{H} 2_{\text {LOS }}^{H} \boldsymbol{H} 2_{L O S}\right)^{-1} \boldsymbol{H} 2_{L O S}^{H}\right] \boldsymbol{H}_{\text {Ray }}
\end{aligned}
$$

Using the inversion matrix lemma $[\boldsymbol{A}+\boldsymbol{B} \boldsymbol{C D}]^{-1}=\boldsymbol{A}^{-1}-\boldsymbol{A}^{-1} \boldsymbol{B}\left[\mathbf{D} \boldsymbol{A}^{-1} \mathbf{B}+\boldsymbol{C}^{-1}\right] \mathbf{D} \boldsymbol{A}^{-1}$

Equation (31) reduces to

$$
\begin{gathered}
\boldsymbol{I}_{N}-\frac{\rho}{N} a^{2} H 1_{L O S}\left(\boldsymbol{I}_{N}+\frac{\rho}{N} a^{2} H 1_{L O S}^{H} H 1_{L O S}\right)^{-1} H 1_{L O S}^{H}=\left(I_{N}+\frac{\rho}{N} a^{2} H 1_{L O S}^{H} H 1_{L O S}\right)^{-1} \\
\boldsymbol{I}_{N}-\frac{\rho}{N} b^{2} H 2_{L O S}\left(\boldsymbol{I}_{N}+\frac{\rho}{N} b^{2} H 2_{L O S}^{H} H 2_{L O S}\right)^{-1} H 2_{L O S}^{H}=\left(\boldsymbol{I}_{N}+\frac{\rho}{N} b^{2} H 2_{L O S}^{H} H 2_{L O S}\right)^{-1}
\end{gathered}
$$

Using (32) and (33), expression (31) can be further reduced to

$$
\begin{aligned}
& I_{N}+\frac{\rho}{N} c^{2} \boldsymbol{H}_{\text {Ray }}^{\boldsymbol{H}}\left(\boldsymbol{I}_{\boldsymbol{N}}+\frac{\rho}{N} a^{2} \boldsymbol{H} \mathbf{1}_{\text {LOS }}^{\boldsymbol{H}} \boldsymbol{H} \mathbf{1}_{L O S}\right)^{-1} \boldsymbol{H}_{\text {Ray }^{+}} \frac{\rho}{N} c^{2} \boldsymbol{H}_{\text {Ray }}^{\boldsymbol{H}}\left(\boldsymbol{I}_{N}+\frac{\rho}{N} b^{2} \boldsymbol{H} \mathbf{2}_{\text {LOS }}^{\boldsymbol{H}} \boldsymbol{H} \mathbf{2}_{L O S}\right)^{-1} \boldsymbol{H}_{\text {Ray }} \\
& \text { Since, } \boldsymbol{I}_{\boldsymbol{N}}+\frac{\rho}{N} a^{2} \boldsymbol{H} \mathbf{1}_{L O S}^{H} \boldsymbol{H} \mathbf{1}_{L O S}>0 \\
& \boldsymbol{I}_{\boldsymbol{N}}+\frac{\rho}{N} b^{2} \boldsymbol{H} \mathbf{2}_{\mathrm{LOS}}^{\mathrm{H}} \boldsymbol{H} \mathbf{2}_{\mathrm{LOS}}>0
\end{aligned}
$$

And $\frac{\rho}{N} c^{2} \boldsymbol{H}_{\boldsymbol{R} a \boldsymbol{y}}^{\boldsymbol{H}} \boldsymbol{H}_{\boldsymbol{R} a \boldsymbol{y}}>0$ then from (34) we have,

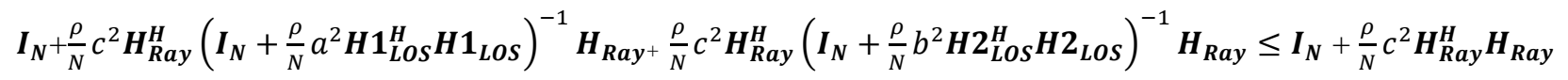

Hence, determinant expression can be given as:-

$$
\text { Det }\{Z\} \leq \operatorname{det}\left\{\boldsymbol{I}_{\boldsymbol{N}}+\frac{\rho}{N} c^{2} \boldsymbol{H}_{\boldsymbol{R} a \boldsymbol{y}}^{\boldsymbol{H}} \boldsymbol{H}_{\boldsymbol{R} a y}\right\}
$$

Now, finally upper bound on the determinant expression can be given as 


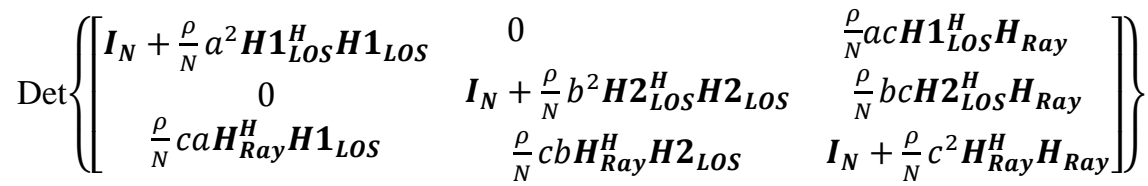

$$
\begin{aligned}
& =\operatorname{det}\left\{\boldsymbol{I}_{\boldsymbol{N}}+\frac{\rho}{N} a^{2} \boldsymbol{H} \mathbf{1}_{\text {Los }}^{\boldsymbol{H}} \boldsymbol{H} \mathbf{1}_{\text {Los }}\right\} \times \operatorname{det}\left\{\boldsymbol{I}_{\boldsymbol{N}}+\frac{\rho}{N} \boldsymbol{a}^{2} \boldsymbol{H} \mathbf{1}_{\boldsymbol{L O S}}^{\boldsymbol{H}} \boldsymbol{H} \mathbf{1}_{\boldsymbol{L O S}}\right\} \times \operatorname{det}\{\mathrm{Z}\} \\
& \leq \operatorname{de}\left\{\boldsymbol{I}_{\boldsymbol{N}}+\frac{\rho}{N} a^{2} \boldsymbol{H} \mathbf{1}_{\boldsymbol{L} \boldsymbol{O} \boldsymbol{S}}^{\boldsymbol{H}} \boldsymbol{H} \mathbf{1}_{\text {Los }}\right\} \times \operatorname{det}\left\{\boldsymbol{I}_{\boldsymbol{N}}+\frac{\rho}{N} a^{2} \boldsymbol{H} \mathbf{1}_{\boldsymbol{L} \boldsymbol{O} \boldsymbol{H}}^{\boldsymbol{H}} \boldsymbol{H} \mathbf{1}_{\boldsymbol{L O S}}\right\} \times \operatorname{det}\left\{\boldsymbol{I}_{\boldsymbol{N}}+\frac{\rho}{N} c^{2} \boldsymbol{H}_{\text {Ray }}^{\boldsymbol{H}} \boldsymbol{H}_{\text {Ray }}\right\}
\end{aligned}
$$

Determinant in capacity expression can be given as

$$
\begin{aligned}
& \left.\operatorname{det}\left(I_{N}+\frac{\rho}{N} a^{2} \boldsymbol{H} \mathbf{1}_{\text {Los }} \boldsymbol{H} \mathbf{1}_{\text {LOS }}^{H}+\frac{\rho}{N} b^{2} \boldsymbol{H} \mathbf{2}_{\text {Los }} \boldsymbol{H} \mathbf{2}_{\text {LOS }}^{H}+c^{2} \boldsymbol{H}_{\text {Ray }} \boldsymbol{H}_{\text {Ray }}^{H}\right) \leq \operatorname{det} I_{N}+\frac{\rho}{N} a^{2} \boldsymbol{H} \mathbf{1}_{\text {Los }} \boldsymbol{H} \mathbf{1}_{\text {Los }}^{H}\right) \times \operatorname{det}\left(I_{N}+\right. \\
& \left.\frac{\rho}{N} \boldsymbol{b}^{\mathbf{2}} \boldsymbol{H} \mathbf{2}_{\text {Los }} \boldsymbol{H} \mathbf{2}_{\text {LOS }}^{H}\right) \times \operatorname{det}\left(I_{N}+\frac{\rho}{N} \boldsymbol{H}_{\text {Ray }} \boldsymbol{H}_{\text {Ray }}^{H}\right)
\end{aligned}
$$

According to work done by authors in [11], an upper bound on logarithmic expression can be put. Since, $\mathrm{f}(\mathrm{x})=\log _{2}(x)$ is strictly increasing function, applying this function on determinant inequality given above generates:-

$$
\begin{aligned}
& \log _{2} \operatorname{det}\left(I_{N}+\frac{\rho}{N} a^{2} \boldsymbol{H} \mathbf{1}_{\text {Los }} \boldsymbol{H} \mathbf{1}_{\text {LOS }}^{H}+\frac{\rho}{N} b^{2} \boldsymbol{H} \mathbf{2}_{\text {Los }} \boldsymbol{H} \mathbf{2}_{\text {LOS }}^{H}+c^{2} \boldsymbol{H}_{\text {Ray }} \boldsymbol{H}_{\text {Ray }}^{H}\right. \\
& \leq \log _{2} \operatorname{det}\left(I_{N}+\frac{\rho}{N} a^{2} \boldsymbol{H} \mathbf{1}_{\text {LoS }} \boldsymbol{H} \mathbf{1}_{\text {LOS }}^{H}\right) \times \operatorname{det}\left(I_{N}+\frac{\rho}{N} b^{2} \boldsymbol{H} \mathbf{2}_{\text {LOS }} \boldsymbol{H} \mathbf{2}_{\text {LOS }}^{H}\right) \times \operatorname{det}\left(I_{N}+\right. \\
& \left.\frac{\rho}{N} \boldsymbol{H}_{\text {Ray }} \boldsymbol{H}_{\text {Ray }}^{H}\right)=\log _{2} \operatorname{det}\left(I_{N}+\frac{\rho}{N} a^{2} \boldsymbol{H} \mathbf{1}_{\text {Los }} \boldsymbol{H} \mathbf{1}_{\text {Los }}^{H}\right)+\log _{2} \operatorname{det}\left(I_{N}+\frac{\rho}{N} b^{2} \boldsymbol{H} \mathbf{2}_{\text {Los }} \boldsymbol{H} \mathbf{2}_{\text {Los }}^{H}\right)+\log _{2} \operatorname{det}\left(I_{N}+\frac{\rho}{N} c^{2} \boldsymbol{H}_{\text {Ray }} \boldsymbol{H}_{\text {Ray }}^{H}\right)
\end{aligned}
$$

Hence upper bound on Capacity of MIMO- TWDP channel can be given as :-

$$
C_{T W D P} \leq C_{L O S 1}+C_{L O S 2}+C_{R a y}
$$

Hence from (15) and (39) it is clear that the capacity of MIMO system always lie between

$$
\mathrm{N} * \log _{2}\left(\frac{\rho}{e}\right) \leq C_{T W D P} \leq C_{L O S 1}+C_{L O S 2}+C_{R a y}
$$

The lower and upper bound of MIMO system in TWDP channel with $N_{t}$ and $N_{r}$ equal to 4 are plotted in Figure 4 . At SNR of $40 \mathrm{~dB}$, maximum capacity attained is $64 \mathrm{bits} / \mathrm{sec} / \mathrm{hertz}$ and lowest capacity that MIMO system can attain in TWDP channel is $51 \mathrm{bits} / \mathrm{sec} / \mathrm{hertz}$. Actual capacity of $4 \times 4$ MIMO system in TWDP channel validates the results as it lies in between upper and lower bound of capacity.

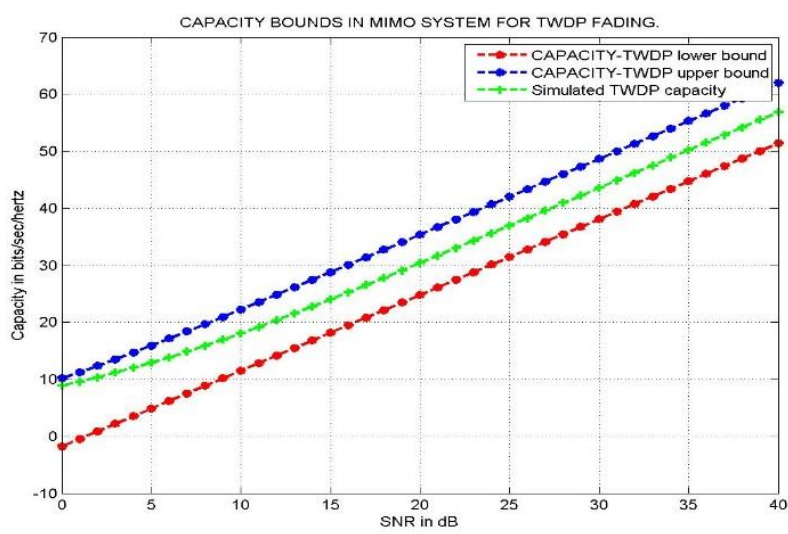

Figure 4. Capacity bounds of MIMO system in TWDP channels. 


\section{Conclusion and Future Scope}

In this paper TWDP channel model for MIMO system have been presented. It is shown that TWDP channel provides a link which is even worse than Rayleigh fading channel. Hence, TWDP channel model can be considered as worst case fading scenario and should be used in testing a wireless communication system in worst case scenario. MIMO systems which use scattering, diffraction and fading in its advantage and attains higher data rate in more faded environment. It is shown through simulations that more capacity is achieved in TWDP fading environment as compared to Rayleigh or Rician fading environment. Two parameters K and $\Delta$ of TWDP fading affect the capacity of MIMO system and by increasing both K and $\Delta$ capacity decreases. Upper and lower bounds on capacity achieved by MIMO in TWDP fading are also derived. TWDP fading model offers new areas of research. Current wireless needs, implementation of wireless sensors for security and maintenance, require that this model is correctly modelled. Its pdf has no close form expression which makes its implementation difficult; hence simple mathematical representation will solve a lot of problems which due to limited time was out of scope of this work. This work has considered performance of MIMO systems in TWDP fading only but Massive MIMO systems too need to be discussed in this fading scenario.

\section{References}

1. Antennas and propagation, http://www.radioelectronics.com/info/antennas/mimo/formats-siso-simo-miso-mimo.php .

2. Rajarajan S, Chaturvedi SK, "On Throughput-Reliability Tradeoff Analysis of MIMO Channels under Generalistic Fading Scenario," International Journal of Performability Engineering. 2017 May;13(3):265.

3. Magleby A, Furse C, "Predicted MIMO performance in intra-vehicle channels," In Antennas and Propagation Society International Symposium, 2008. AP-S 2008. IEEE 2008 Jul 5 (pp. 1-4). IEEE.

4. Oh SH, Li KH, "BER performance of BPSK receivers over two-wave with diffuse power fading channels," IEEE Transactions on Wireless Communications. 2005 Jul;4(4):1448-54.

5. Zhen H, "Performance evaluation of 2-D pilot aided OFDM system under hyper-Rayleigh fading channel," (Doctoral dissertation, University of Toledo).

6. Durgin GD, Rappaport TS, De Wolf DA, "New analytical models and probability density functions for fading in wireless communications," IEEE Transactions on Communications. 2002 Jun;50(6):1005-15.

7. Frolik J, "On appropriate models for characterizing hyper-Rayleigh fading," IEEE Transactions on Wireless Communications. 2008 Dec;7(12).

8. Rao M, Lopez-Martinez FJ, Alouini MS, Goldsmith A, "MGF approach to the analysis of generalized two-ray fading models," IEEE Transactions on Wireless Communications. 2015 May;14(5):2548-61.

9. Foschini GJ, Gans MJ, "On limits of wireless communications in a fading environment when using multiple antennas," Wireless Personal Communications. 1998 Mar 1;6(3):311-35.

10. Dong W, Li J, Lu Z, "Parameter estimation for correlated MIMO channels with frequency-selective fading," Wireless Personal Communications. 2010 Mar 1;52(4):813-28.

11. Ayadi J, Hutter AA, Farserotu J, "On the multiple input multiple output capacity of Rician channels," In Wireless Personal Multimedia Communications, 2002. The 5th International Symposium on 2002 Oct 27 (Vol. 2, pp. 402-406). IEEE.

Bhavnika Garg is working as an assistant professor in Department of Electrical and Electronics Engineering at Lovely Professional University, Jalandhar. She has two years of teaching and research experience. Currently, she is working in the area of Wireless Communication, MIMO systems, Massive MIMO systems and spatial Modulation.

Aman Singh is working as an assistant professor in Department of Computer Science and Engineering at Lovely Professional University, Punjab, India. He has five years of teaching and research experience and his areas of interest are wireless communication, adhoc networks, biomedical engineering, information security, cyber-crime and computer forensics. 